\title{
A GENERALIZATION OF THE CASSELS-TATE DUAL EXACT SEQUENCE
}

\author{
Cristian D. GonzÁlez-Avilés and Ki-Seng Tan
}

\begin{abstract}
We extend the first part of the well-known Cassels-Tate dual exact sequence for abelian varieties $A$ over global fields $K$ in two directions: we treat the $p$-primary component in the function field case, where $p$ is the characteristic of $K$, and we dispense with the assumption that the Tate-Shafarevich group of $A$ is finite.
\end{abstract}

\section{Introduction}

Let $K$ be a global field and let $m$ be a positive integer which is prime to the characteristic of $K$ (in the function field case). Let $A$ be an abelian variety over $K$. Then there exists an exact sequence of discrete groups

$$
0 \rightarrow \amalg(A)(m) \rightarrow H^{1}(K, A)(m) \rightarrow \bigoplus_{\text {all } v} H^{1}\left(K_{v}, A\right)(m) \rightarrow \mathrm{Б}(A)(m) \rightarrow 0,
$$

where $K_{v}$ is the henselization of $K$ at $v, M(m)$ denotes the $m$-primary component of a torsion abelian group $M$, and $\mathrm{E}(A)$ is defined to be the cokernel of the localization map $H^{1}(K, A) \rightarrow \bigoplus_{\text {all } v} H^{1}\left(K_{v}, A\right)$. The Pontrjagyn dual of the preceding exact sequence is an exact sequence of compact groups

$$
0 \leftarrow \amalg(A)(m)^{*} \leftarrow H^{1}(K, A)(m)^{*} \leftarrow \prod_{\text {all } v} H^{0}\left(K_{v}, A^{t}\right)^{\prec} \leftarrow \mathrm{Б}(A)(m)^{*} \leftarrow 0,
$$

where $A^{t}$ is the abelian variety dual to $A$ and, for any abelian group $M, M^{\wedge}$ denotes the $m$-adic completion $\lim _{n} M / m^{n}$ of $M$. Now, if $\amalg(A)(m)$ is finite (or, more generally, if $\amalg(A)(m)$ contains no nontrivial elements which are divisible by $m^{n}$ for every $n \geq 1)$, then $\amalg(A)(m)^{*}$ and $\mathrm{E}(A)(m)^{*}$ are canonically isomorphic to $\amalg\left(A^{t}\right)(m)$ and $A^{t}(K)^{\uparrow}$, respectively, and the preceding exact sequence induces an exact sequence

$$
0 \leftarrow \amalg\left(A^{t}\right)(m) \leftarrow H^{1}(K, A)(m)^{*} \leftarrow \prod_{\text {all } v} H^{0}\left(K_{v}, A^{t}\right)^{\wedge} \leftarrow A^{t}(K)^{\wedge} \leftarrow 0
$$

which is known as the Cassels-Tate dual exact sequence. See [9, Theorem II.5.6(b), p.247]. The aim of this paper is to extend the first part of the above exact sequence

Received by the editors August 23, 2006.

2000 Mathematics Subject Classification. Primary 11G35; Secondary 14 G25.

Key words and phrases. Selmer groups, Tate-Shafarevich groups, Cassels-Tate dual exact sequence.

C.G.-A. is partially supported by Fondecyt grant 1061209 and Universidad Andrés Bello grant DI-29-05/R.

K.-S.T. is partially supported by the National Science Council of Taiwan, NSC91-2115-M-002-001, NSC94-2115-M-002-010. 
to the case where $m$ is divisible by the characteristic of $K$ (in the function field case) and no hypotheses are made on $\amalg(A)$. The following is the main result of the paper. Let $m$ and $n$ be arbitrary positive integers. Set

$$
\operatorname{Sel}\left(A^{t}\right)_{m^{n}}=\operatorname{Ker}\left[H^{1}\left(K, A_{m^{n}}^{t}\right) \rightarrow \bigoplus_{\text {all } v} H^{1}\left(K_{v}, A^{t}\right)\right]
$$

and

$$
T_{m} \operatorname{Sel}\left(A^{t}\right)=\underbrace{\lim }_{n} \operatorname{Sel}\left(A^{t}\right)_{m^{n}}
$$

Then the following holds ${ }^{1}$ :

Main Theorem. For any positive integer $m$, there exists a natural exact sequence of compact groups

$$
0 \leftarrow \amalg(A)(m)^{*} \leftarrow H^{1}(K, A)(m)^{*} \leftarrow \prod_{\text {all } v} H^{0}\left(K_{v}, A^{t}\right)^{\curlywedge} \leftarrow T_{m} \operatorname{Sel}\left(A^{t}\right) \leftarrow 0 .
$$

We should note that a similar statement holds true if above the henselizations of $K$ are replaced by its completions. See [9, Remark I.3.10, p.59].

This paper grew out of questions posed to the authors by B.Poonen, in connection with the forthcoming paper [11]. We expect that the above theorem will be useful in [op.cit.].

\section{Settings and notations}

Let $K$ be a global field and let $A$ be an abelian variety over $K$. In the function field case, we let $p$ denote the characteristic of $K$. All cohomology groups below are either Galois cohomology groups or flat cohomology groups. For any non-archimedean prime $v$ of $K, K_{v}$ will denote the field of fractions of the henselization of the ring of $v$-integers of $K$. If $v$ is an archimedean prime, $K_{v}$ will denote the completion of $K$ at $v$, and we will write $H^{0}\left(K_{v}, A\right)$ for the quotient of $A\left(K_{v}\right)$ by its identity component. Note that, for any prime $v$ of $K$, the group $H^{1}\left(K_{v}, A\right)$ is canonically isomorphic to $H^{1}\left(\widehat{K}_{v}, A\right)$, where $\widehat{K}_{v}$ denotes the completion of $K$ at $v$. See [9, Remark I.3.10(ii), p.58]. Now let $X$ denote either the spectrum of the ring of integers of $K$ (in the number field case) or the unique smooth complete curve over the field of constants of $K$ with function field $K$ (in the function field case). In what follows, $U$ denotes a nonempty open subset of $X$ such that $A$ has good reduction over $U$. When $N$ is a quasi-finite flat group scheme on $U$, we endow $H^{r}(U, N)$ with the discrete topology. Now let $m$ and $n$ be arbitrary positive integers, and let $M$ be an abelian topological group. We will write $M / m^{n}$ for $M / m^{n} M=M \otimes_{\mathbb{Z}} \mathbb{Z} / m^{n}$ and $M^{\curlywedge}$ for the $m$-adic completion $\varliminf_{n} M / m^{n}$ of $M$. Further, we set $\mathbb{Z}_{m}=\prod_{\ell \mid m} \mathbb{Z}_{\ell}, \mathbb{Q}_{m}=\mathbb{Z}_{m} \otimes_{\mathbb{Z}} \mathbb{Q}$ and define $M^{*}=\operatorname{Hom}_{\text {cts }}\left(M, \mathbb{Q}_{m} / \mathbb{Z}_{m}\right)$. Finally, the $m$-primary component of a torsion group $M$ will be denoted by $M(m)$.

\footnotetext{
${ }^{1}$ To see why the exact sequence of the theorem extends the Cassels-Tate dual exact sequence recalled above, see exact sequence (6) below, note that $T_{m} \amalg(A)=T_{m}\left(\amalg(A)_{m \text {-div }}\right)$ vanishes if $\amalg(A)_{m \text {-div }}=0$, and use $[9$, Corollary III.9.5, p.370].
} 


\section{Proof of the Main Theorem}

Both $A$ and its dual variety $A^{t}$ extend to abelian schemes $\mathcal{A}$ and $\mathcal{A}^{t}$ over $U$ (see [2, Ch.1, §1.4.3]). By [5, VIII.7.1(b)], the canonical Poincaré biextension of $\left(A^{t}, A\right)$ by $\mathbb{G}_{m}$ extends to a biextension over $U$ of $\left(\mathcal{A}^{t}, \mathcal{A}\right)$ by $\mathbb{G}_{m}$. Further, by [op.cit., VII.3.6.5], (the isomorphism class of) this biextension corresponds to a map $\mathcal{A}^{t} \otimes \mathbf{L} \mathcal{A} \rightarrow \mathbb{G}_{m}[1]$ in the derived category of the category of smooth sheaves on $U$. This map in turn induces (see $\left[9\right.$, p.283]) a canonical pairing $H^{1}\left(U, \mathcal{A}^{t}\right) \times H_{\mathrm{c}}^{1}(U, \mathcal{A}) \rightarrow H_{\mathrm{c}}^{3}\left(U, \mathbb{G}_{m}\right) \simeq \mathbb{Q} / \mathbb{Z}$, where the $H_{\mathrm{c}}^{r}(U, \mathcal{A})$ are the cohomology groups with compact support of the sheaf $\mathcal{A}$ defined in $[9$, p.271].

Remark 3.1. The smoothness of $\mathcal{A}$ implies that the groups $H^{r}(U, \mathcal{A})$ and $H_{\mathrm{c}}^{r}(U, \mathcal{A})$ agree with the analogous groups defined for the étale topology. See $[9$, Proposition III.0.4(d), p.272].

For any positive integer $m$ and any $n \geq 1$, the above pairing induces a pairing

$$
H^{1}\left(U, \mathcal{A}_{m^{n}}^{t}\right) \times H_{\mathrm{c}}^{1}(U, \mathcal{A}) / m^{n} \rightarrow \mathbb{Q} / \mathbb{Z} .
$$

On the other hand, the map $\mathcal{A}^{t} \otimes \mathbf{L} \mathcal{A} \rightarrow \mathbb{G}_{m}[1]$ canonically defines a map $\mathcal{A}_{m^{n}}^{t} \times \mathcal{A}_{m^{n}} \rightarrow$ $\mathbb{G}_{m}$, which induces a pairing

$$
H^{1}\left(U, \mathcal{A}_{m^{n}}^{t}\right) \times H_{\mathrm{c}}^{2}\left(U, \mathcal{A}_{m^{n}}\right) \rightarrow \mathbb{Q} / \mathbb{Z} .
$$

The preceding pairing induces an isomorphism

$$
H_{\mathrm{c}}^{2}\left(U, \mathcal{A}_{m^{n}}\right) \stackrel{\sim}{\longrightarrow} H^{1}\left(U, \mathcal{A}_{m^{n}}^{t}\right)^{*} .
$$

See [9, Corollary II.3.3, p.217] for the case where $m$ prime to $p$, and [op.cit., Theorem III.8.2, p.361] for the case where $m$ is divisible by $p$. The pairings (1) and (2) are compatible, in the sense that the following diagram commutes:

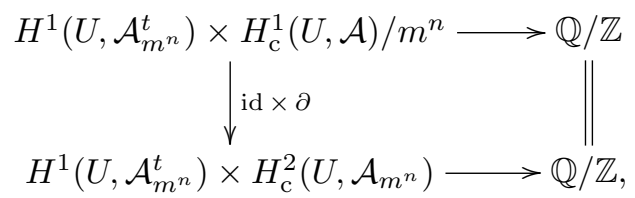

where $\partial: H_{\mathrm{c}}^{1}(U, \mathcal{A}) / m^{n} \hookrightarrow H_{\mathrm{c}}^{2}\left(U, \mathcal{A}_{m^{n}}\right)$ is induced by the connecting homomorphism $H_{\mathrm{c}}^{1}(U, \mathcal{A}) \rightarrow H_{\mathrm{c}}^{2}\left(U, \mathcal{A}_{m^{n}}\right)$ coming from the exact sequence

$$
0 \rightarrow \mathcal{A}_{m^{n}} \rightarrow \mathcal{A} \stackrel{m^{n}}{\rightarrow} \mathcal{A} \rightarrow 0 .
$$

Now define

$$
\operatorname{Sel}\left(A^{t}\right)_{m^{n}}=\operatorname{Ker}\left[H^{1}\left(K, A_{m^{n}}^{t}\right) \rightarrow \bigoplus_{\text {all } v} H^{1}\left(K_{v}, A^{t}\right)\right]
$$

and

$$
T_{m} \operatorname{Sel}\left(A^{t}\right)=\underbrace{\lim }_{n} \operatorname{Sel}\left(A^{t}\right)_{m^{n}}
$$

Now the kernel-cokernel sequence of the pair of maps $H^{1}\left(K, A_{m^{n}}^{t}\right) \rightarrow H^{1}\left(K, A^{t}\right)_{m^{n}} \rightarrow$ $\bigoplus_{\text {all } v} H^{1}\left(K_{v}, A^{t}\right)$ [9, Proposition I.0.24, p.19] yields an exact sequence

$$
0 \rightarrow A^{t}(K) / m^{n} \rightarrow \operatorname{Sel}\left(A^{t}\right)_{m^{n}} \rightarrow \amalg\left(A^{t}\right)_{m^{n}} \rightarrow 0 .
$$


Taking inverse limits, we obtain an exact sequence

$$
0 \rightarrow A^{t}(K)^{\wedge} \rightarrow T_{m} \operatorname{Sel}\left(A^{t}\right) \rightarrow T_{m} \amalg\left(A^{t}\right) \rightarrow 0 .
$$

See [1, Proposition 10.2, p.104]. Now define ${ }^{2}$

$$
D^{1}\left(U, \mathcal{A}_{m^{n}}^{t}\right)=\operatorname{Ker}\left[H^{1}\left(U, \mathcal{A}_{m^{n}}^{t}\right) \rightarrow \prod_{v \notin U} H^{1}\left(K_{v}, A^{t}\right)\right]
$$

and

$$
\begin{aligned}
D^{1}\left(U, \mathcal{A}^{t}\right) & =\operatorname{Im}\left[H_{\mathrm{c}}^{1}\left(U, \mathcal{A}^{t}\right) \rightarrow H^{1}\left(U, \mathcal{A}^{t}\right)\right] \\
& =\operatorname{Ker}\left[H^{1}\left(U, \mathcal{A}^{t}\right) \rightarrow \prod_{v \notin U} H^{1}\left(K_{v}, A^{t}\right)\right] .
\end{aligned}
$$

Note that the pairing $H^{1}\left(U, \mathcal{A}^{t}\right) \times H_{\mathrm{c}}^{1}(U, \mathcal{A}) \rightarrow \mathbb{Q} / \mathbb{Z}$ induces a pairing $D^{1}\left(U, \mathcal{A}^{t}\right) \times$ $D^{1}(U, \mathcal{A}) \rightarrow \mathbb{Q} / \mathbb{Z}$

By $[9$, Proposition III.0.4(a), p.271] and the right-exactness of the tensor product functor, there exists a natural exact sequence

$$
\bigoplus_{v \notin U} H^{0}\left(K_{v}, A\right) / m^{n} \rightarrow H_{\mathrm{c}}^{1}(U, \mathcal{A}) / m^{n} \rightarrow D^{1}(U, \mathcal{A}) / m^{n} \rightarrow 0 .
$$

Lemma 3.2. The map $H^{1}\left(U, \mathcal{A}_{m^{n}}^{t}\right) \hookrightarrow H^{1}\left(K, A_{m^{n}}^{t}\right)$ induces an isomorphism

$$
D^{1}\left(U, \mathcal{A}_{m^{n}}^{t}\right) \simeq \operatorname{Sel}\left(A^{t}\right)_{m^{n}}
$$

Proof. By [9, Lemma II.5.5, p.246] and Remark 3.1 above, the map $H^{1}\left(U, \mathcal{A}^{t}\right) \hookrightarrow$ $H^{1}\left(K, A^{t}\right)$ induces an isomorphism

$$
D^{1}\left(U, \mathcal{A}^{t}\right)_{m^{n}} \simeq \amalg\left(A^{t}\right)_{m^{n}} .
$$

Now $H^{1}\left(U, \mathcal{A}_{m^{n}}^{t}\right) \rightarrow \prod_{v \in U} H^{1}\left(K_{v}, A^{t}\right)$ factors through $H^{1}\left(U, \mathcal{A}^{t}\right) \rightarrow \prod_{v \in U} H^{1}\left(K_{v}, A^{t}\right)$, which is the zero map (see $[9,(5.5 .1)$, p.247] and Remark 3.1 above). Consequently, $H^{1}\left(U, \mathcal{A}_{m^{n}}^{t}\right) \hookrightarrow H^{1}\left(K, A_{m^{n}}^{t}\right)$ maps $D^{1}\left(U, \mathcal{A}_{m^{n}}^{t}\right)$ into $\operatorname{Sel}\left(A^{t}\right)_{m^{n}}$. To prove surjectivity, we consider the commutative diagram

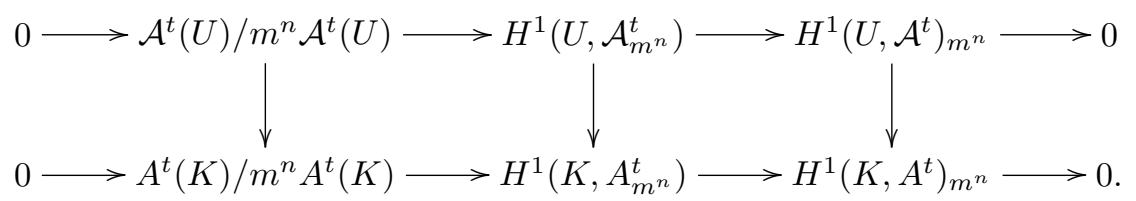

Note that the properness of $\mathcal{A}^{t}$ over $U$ implies that the left-hand vertical map in the above diagram is an isomorphism (see [op.cit., p.242]). Now let $c \in \operatorname{Sel}\left(A^{t}\right)_{m^{n}}$, write $c^{\prime}$ for its image in $\amalg\left(A^{t}\right)_{m^{n}}$ under the map in (5) and let $\xi^{\prime} \in D^{1}\left(U, \mathcal{A}^{t}\right)_{m^{n}} \subset$ $H^{1}\left(U, \mathcal{A}^{t}\right)_{m^{n}}$ be the pullback of $c^{\prime}$ under the isomorphism $D^{1}\left(U, \mathcal{A}^{t}\right)_{m^{n}} \simeq \amalg\left(A^{t}\right)_{m^{n}}$ recalled above. Then the fact that the left-hand vertical map in the above diagram is an isomorphism implies that $\xi^{\prime}$ can be pulled back to a class $\xi \in H^{1}\left(U, \mathcal{A}_{m^{n}}^{t}\right)$ which maps down to $c$. Clearly $\xi \in D^{1}\left(U, \mathcal{A}_{m^{n}}^{t}\right)$, and this completes the proof.

The following proposition generalizes [9, Theorem II.5.2(c), p.244].

\footnotetext{
${ }^{2}$ In these definitions, the products extend over all primes of $K$, including the archimedean primes, not in $U$.
} 
Proposition 3.3. There exists a canonical isomorphism

$$
\left(T_{m} \operatorname{Sel}\left(A^{t}\right)\right)^{*} \stackrel{\sim}{\longrightarrow} H_{\mathrm{c}}^{2}(U, \mathcal{A})(m) .
$$

Proof. There exists a commutative diagram

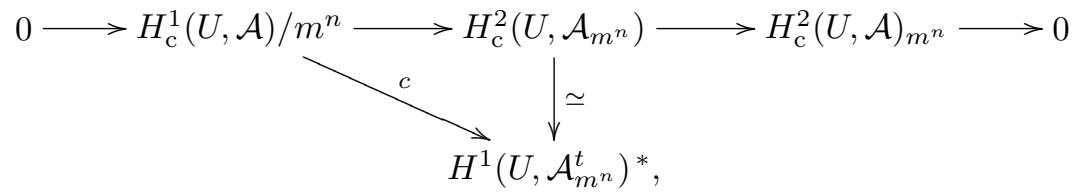

where the vertical map is the isomorphism (3). Clearly, the above diagram induces an isomorphism Coker $c \simeq H_{\mathrm{c}}^{2}(U, \mathcal{A})_{m^{n}}$. On the other hand, there exists a natural exact commutative ${ }^{3}$ diagram

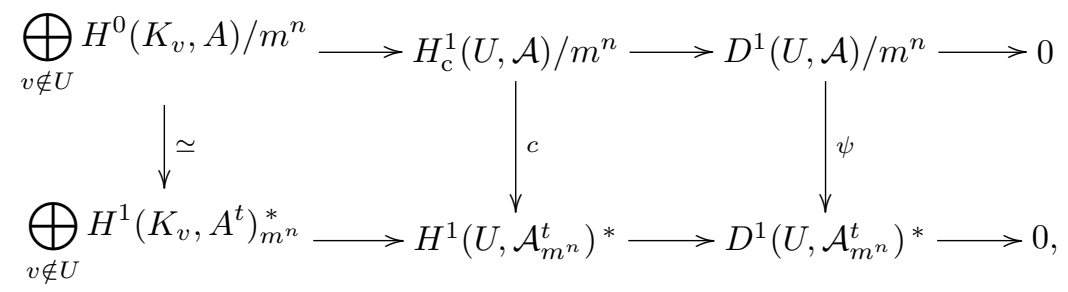

where the top row is (7), the right-hand vertical map $\psi$ is the composite of the natural map $D^{1}(U, \mathcal{A}) / m^{n} \rightarrow D^{1}\left(U, \mathcal{A}^{t}\right)_{m^{n}}^{*}$ induced by the pairing $D^{1}\left(U, \mathcal{A}^{t}\right) \times D^{1}(U, \mathcal{A}) \rightarrow$ $\mathbb{Q} / \mathbb{Z}$ and the natural map $D^{1}\left(U, \mathcal{A}^{t}\right)_{m^{n}}^{*} \rightarrow D^{1}\left(U, \mathcal{A}_{m^{n}}^{t}\right)^{*}$, and the left-hand vertical map is induced by the canonical Poincaré biextensions of $\left(A^{t}, A\right)$ by $\mathbb{G}_{m}$ over $K_{v}$ for each $v \notin U$. That the latter map is an isomorphism follows from [9, Remarks I.3.5 and I.3.7, pp.53 and 56, and Theorem III.7.8, p.354] and the fact that the pairings defined in [loc.cit.] are compatible with the pairing induced by the canonical Poincaré biextension (see [4, Appendix]). The above diagram and the identification Coker $c=H_{\mathrm{c}}^{2}(U, \mathcal{A})_{m^{n}}$ yield an exact sequence

$$
D^{1}(U, \mathcal{A}) / m^{n} \rightarrow D^{1}\left(U, \mathcal{A}_{m^{n}}^{t}\right)^{*} \rightarrow H_{\mathrm{c}}^{2}(U, \mathcal{A})_{m^{n}} \rightarrow 0
$$

Taking direct limits, we obtain an exact sequence

$$
D^{1}(U, \mathcal{A}) \otimes \mathbb{Q}_{m} / \mathbb{Z}_{m} \rightarrow\left(\lim _{\longleftarrow} D^{1}\left(U, \mathcal{A}_{m^{n}}^{t}\right)\right)^{*} \rightarrow H_{\mathrm{c}}^{2}(U, \mathcal{A})(m) \rightarrow 0
$$

But $D^{1}(U, \mathcal{A}) \otimes \mathbb{Q}_{m} / \mathbb{Z}_{m}=0$ since $D^{1}(U, \mathcal{A})$ is torsion and $\mathbb{Q}_{m} / \mathbb{Z}_{m}$ is divisible. Now lemma 3.2 completes the proof.

By Remark 3.1 and [9, proof of Lemma II.5.5, p.247, and Proposition II.2.3, p. 203], there exist exact sequences ${ }^{4}$

$$
H^{1}(U, \mathcal{A}) \stackrel{c_{U}}{\longrightarrow} \bigoplus_{v \notin U} H^{1}\left(K_{v}, A\right) \rightarrow H_{\mathrm{c}}^{2}(U, \mathcal{A})
$$

and

$$
0 \rightarrow H^{1}(U, \mathcal{A}) \stackrel{i_{U}}{\longrightarrow} H^{1}(K, A) \stackrel{\lambda_{U}}{\longrightarrow} \bigoplus_{v \in U} H^{1}\left(K_{v}, A\right)
$$

\footnotetext{
${ }^{3}$ The commutativity of this diagram follows from that of diagram (4).

${ }^{4}$ In the second exact sequence, " $v \in U$ " is shorthand for " $v$ is a closed point of $U$ ".
} 
where $c_{U}$ and $\lambda_{U}$ are natural localization maps and $i_{U}$ is induced by the inclusion Spec $K \hookrightarrow U$. If $U \subset V$ is an inclusion of nonempty open subsets of $X$, then there exists a natural commutative diagram

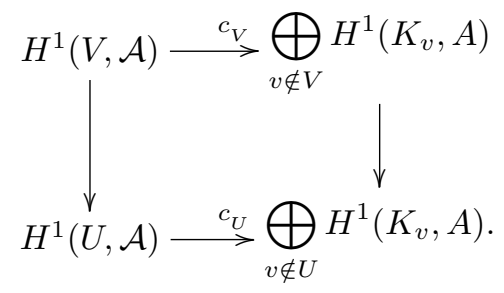

Define

$$
\mathrm{Б}(A)_{U}=\operatorname{coker}\left[c_{U}: H^{1}(U, \mathcal{A}) \rightarrow \bigoplus_{v \notin U} H^{1}\left(K_{v}, A\right)\right],
$$

which we regard as a subgroup of $H_{\mathrm{c}}^{2}(U, \mathcal{A})$. The preceding diagram shows that an inclusion $U \subset V$ of nonempty open subsets of $X$ induces a map $\mathrm{Б}(A)_{V} \rightarrow \mathrm{Б}(A)_{U}$. Define

$$
\mathrm{E}(A)=\lim _{\longrightarrow} \mathrm{S}(A)_{U}=\operatorname{coker}\left[H^{1}(K, A) \rightarrow \bigoplus_{\text {all } v} H^{1}\left(K_{v}, A\right)\right],
$$

where the limit is taken over the directed family of all nonempty open subsets $U$ of $X$ such that $A$ has good reduction over $U$, ordered by $V \leq U$ if and only if $U \subset V$. For each $U$ as above and every $n \geq 1$, there exists an exact sequence

$$
\bigoplus_{v \notin U} H^{1}\left(K_{v}, A\right)_{m^{n}} \rightarrow\left(\mathrm{E}(A)_{U}\right)_{m^{n}} \rightarrow\left(\operatorname{Im} c_{U}\right) / m^{n} .
$$

Since $\operatorname{Im} c_{U}$ is torsion, we conclude that there exists a surjection

$$
\bigoplus_{v \notin U} H^{1}\left(K_{v}, A\right)(m) \longrightarrow \mathrm{Б}(A)_{U}(m) .
$$

On the other hand, there exists a natural injection $T_{m} \operatorname{Sel}\left(A^{t}\right) \hookrightarrow \prod_{\text {all } v} H^{0}\left(K_{v}, A^{t}\right)^{\wedge}$ (when $m$ is prime to $p$, this is contained in [9, Corollary I.6.23(b), p.111]. If $m$ is divisible by $p$, the assertion follows from the $p$-analogue of Proposition I.6.19 of [op.cit.], which in turn follows from $[10]^{5}$. Consequently, there is a surjection

$$
\bigoplus_{\text {all } v}\left(H^{0}\left(K_{v}, A^{t}\right)^{\wedge}\right)^{*} \rightarrow\left(T_{m} \operatorname{Sel}\left(A^{t}\right)\right)^{*}
$$

Further, as noted in the proof of Proposition 3.3, the canonical Poincaré biextensions induce an isomorphism

$$
\bigoplus_{\text {all } v}\left(H^{0}\left(K_{v}, A^{t}\right)^{\wedge}\right)^{*} \simeq \bigoplus_{\text {all } v} H^{1}\left(K_{v}, A\right)(m)
$$

\footnotetext{
${ }^{5}$ See, especially, Propositions 5 and 6 of [10], which extend the results on pp.773 and 734 of [12] to the case $p \mid m$.
} 
whence there exists a surjection

$$
\bigoplus_{\text {all } v} H^{1}\left(K_{v}, A\right)(m) \longrightarrow\left(T_{m} \operatorname{Sel}\left(A^{t}\right)\right)^{*} .
$$

The maps (8) and (9) fit into a commutative diagram

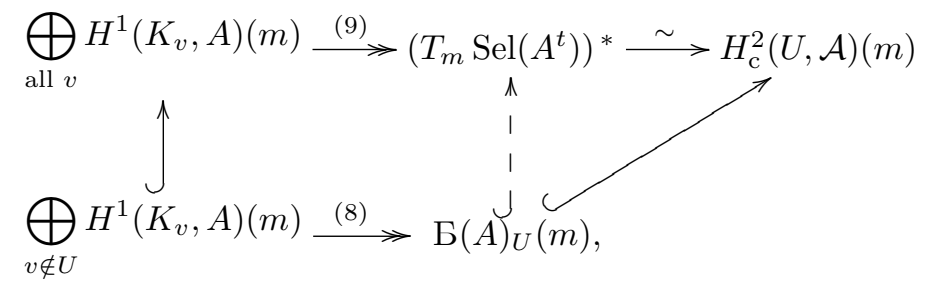

where the isomorphism on the top row exists by Proposition 3.3. Taking the direct limit over $U$ in the above diagram, we conclude that there exists an isomorphism

$$
\mathrm{B}(A)(m) \stackrel{\sim}{\longrightarrow}\left(T_{m} \operatorname{Sel}\left(A^{t}\right)\right)^{*},
$$

as desired.

Remark 3.4. Recently (see [7, Theorem 1.2]), the Cassels-Tate dual exact sequence has been extended to 1-motives $M$ over number fields, under the assumption that the Tate-Shafarevich group of $M$ is finite. Using [6, Remark 5.10], it should not be difficult to extend this result to global function fields, provided the $p$-primary components of the groups involved are ignored. In this paper we have managed to remove the latter restriction when $M$ is an abelian variety, but the problem remains for general 1-motives $M$.

\section{Acknowledgements}

K.-S.T. thanks J.Milne for the helpful outline [8] which led to a complete proof of the $p$-primary part of the Main Theorem of this paper under the assumption that $\amalg(A)$ is finite. C.G.-A. thanks B.Poonen for suggesting Proposition 3.3 and, more generally, for suggesting that the above finiteness assumption can be dispensed with in the relevant proofs of [9] if $A(K)^{\wedge}$ is replaced with $T_{m} \operatorname{Sel}(A)$ throughout $^{6}$. Finally, both authors thank the referee for corrections to the exposition.

\section{References}

[1] M. Atiyah and I. MacDonald, Introduction to Commutative Algebra, Addison-Wesley, 1969.

[2] S. Bosch, W. Lütkebohmert, and M. Raynaud, Néron Models, Springer Verlag, 1990.

[3] J. W. S. Cassels, Arithmetic on curves of genus 1. VII. The dual exact sequence, J. Reine Angew. Math. 216, 1964, 150-158.

[4] C. D. González-Avilés, Brauer groups and Tate-Shafarevich groups, J. Math. Sci. Univ. Tokyo 10, (2003), no. 2, 391-419.

[5] A. Grothendieck, Groupes de Monodromie en Géométrie Algébrique I, Séminaire de Géométrie Algébrique du Bois Marie 1967-69 (SGA 7 I), Lecture Notes in Math., 288, Springer, 1972.

\footnotetext{
${ }^{6}$ After this paper was completed, we learned that the existence of a natural duality between $\mathrm{Б}(A)(m)$ and $T_{m} \operatorname{Sel}\left(A^{t}\right)$ had already been observed by Cassels in the case of elliptic curves over number fields. See [3, p.153]. Therefore, the Main Theorem of this paper may be regarded as a natural generalization of Cassels' result.
} 
[6] D. Harari and T. Szamuely, Arithmetic duality theorems for 1-motives, J. Reine Angew. Math. 578, (2005), 93-128.

[7] http://www.renyi.hu/ szamuely.

[8] J. S. Milne, a letter to K.-S.Tan, March 19th, 1991.

[9] _ Arithmetic Duality Theorems, Persp. in Math., 1 Academic Press, 1986.

[10] _ Congruence subgroups of abelian varieties, Bull. Sci. Math. 96 (1972), 333-338.

[11] B. Poonen and F. Voloch, The Brauer-Manin obstruction for subvarieties of abelian varieties over function fields, In preparation.

[12] J.-P. Serre, Sur les groupes de congruence des variétés abéliennes II, Izv. Akad. Nauk SSSR Ser. Mat. 35, (1971), 731-735.

Departamento de Matemáticas, Universidad Andrés Bello, Chile

E-mail address: cristiangonzalez@unab.cl

Department of Mathematics, National Taiwan University, Taipei 106, Taiwan

E-mail address: tan@math.ntu.tw 